\title{
Retos ambientales para un país en vías de desarrollo
}

\author{
Enjoli Matallana, Harold Esenarro, Mario León, David Quispe, \\ Adriana Vallejos y Ricardo Villalobos ${ }^{1}$
}

\section{RESUMEN}

Han pasado ya más de dos décadas desde que se firmó la Declaración de Río sobre el Medio Ambiente y el Desarrollo, y, sin embargo, los esfuerzos alcanzados por los países adscritos no han sido suficientes. En el presente artículo, se reflexiona sobre algunos de los problemas que enfrenta la adecuada protección del medio ambiente, y algunas posibles soluciones desde una perspectiva legal complementada con conceptos básicos de ecología.

\section{PALABRAS CLAVE}

Medio ambiente, legislación ambiental, cambio climático.

\section{ABSTRACT}

It's been over two decades since the Rio Declaration on Environment and Development was signed, and yet the efforts made by the affiliated countries have not been sufficient. In this article, we examine some of the issues facing the proper protection of the environment, and some possible solutions from a legal perspective complemented basics of ecology.

\section{KEY WORDS}

Environment, environmental law, climate change. 


\section{El antropocentrismo y el medio ambiente como sujeto de derecho}

La visión moderna de la naturaleza nos sugiere que esta existe y persiste para beneficio del hombre; lo que es más, su valoración se determina en función de los beneficios que le proporcione y su preservación es relevante en el sentido que pone en peligro la existencia del ser humano, al sustraerle su nicho ecológico. Esto responde a la postura completamente antropocéntrica que se concibe en la actualidad sobre el ambiente ${ }^{2}$ y sobre la cual se desarrolla la gran mayoría de políticas medioambientales existentes a nivel mundial. Sin embargo, desde 1972, con la Declaración de la Conferencia de las Naciones Unidas sobre el Medio Ambiente, se reconoce la necesidad que tiene el hombre de generar políticas para la preservación del medio ambiente, y que es concebido como parte del medio ambiente humano, esencial para su bienestar y el goce de sus derechos fundamentales ${ }^{3}$.

En ese sentido, se incluyeron dentro de esta declaración diferentes principios que, entre otras cosas, introducen por primera vez la necesidad de preservar, proteger y administrar adecuadamente los recursos naturales, en beneficio de las generaciones presentes y futuras ${ }^{4}$. Esto compromete a los países adscritos a dicha declaración a administrar de manera sostenible sus recursos con miras al futuro. No obstante, estas acciones, aunque bien intencionadas, no han tenido un efecto mayor que el compromiso de los Estados a mejorar sus políticas y medidas de protección de acuerdo con sus «circunstancias nacionales» ${ }^{5}$. En este sentido, puede decirse que la legislación medioambiental internacional que hasta ahora se maneja carece del suficiente vigor institucional y legal para su aplicación -lo que en el argot legal anglosajón es bien llamado soft law, que hace alusión a un derecho blando, poco vinculante, de naturaleza incierta-, debido a que solo prevé una sanción de carácter moral como consecuencia de su incumplimiento ${ }^{6}$.

En esta línea de pensamiento, no es sorpresa encontrar, dentro de nuestra propia legislación, fallos que permitan que se siga afectando al medio ambiente de manera irreversible, como el caso del artículo 10 del

\footnotetext{
2 Bedón s.f.

3 Declaración de las Naciones Unidas sobre el Medio Ambiente. En http://www.juridicas.unam. mx/publica/librev/rev/derhum/cont/13/pr/pr23.pdf. Consultado el 6 de junio de 2011.

4 Declaración de las Naciones Unidas sobre el Medio Ambiente. En http://www.juridicas.unam. mx/publica/librev/rev/derhum/cont/13/pr/pr23.pdf. Consultado el 6 de junio de 2011.

5 Naciones Unidas. Protocolo de Kioto de la Convención Marco de las Naciones Unidas sobre el Cambio Climático.

6 European Comission. En http://ec.europa.eu/translation/bulletins/puntoycoma/63/pyc633.htm. Consultado el 24 de junio de 2011.
} 
Decreto Supremo 014-92-EM, Texto Único Ordenado de la Ley General de Minería, que reconoce que las concesiones son un bien intangible, otorgadas de manera irrevocable siempre y cuando el titular cumpla con los requisitos de vigencia, los cuales consisten en los pagos respectivos al Estado por dicho derecho y la presentación de la documentación pertinente, sin considerar cláusulas que propicien su revocación por incumplimientos medioambientales ${ }^{7}$.

El poco vigor jurídico de los tratados internacionales se aúna al hecho de que la manera más vinculante y efectiva de defender el medio ambiente en un ámbito jurídico es por medio de la defensa de nuestro propio derecho a gozar de un ambiente equilibrado y adecuado al desarrollo de nuestras vidas, al bienestar y a la protección de la salud, por cuanto estos son derechos fundamentales inherentes al ser humano, en concordancia con lo expresado en el artículo primero de nuestra actual Carta Magna, que recoge que la defensa de la persona humana y el respeto a su integridad es el fin supremo del Estado.

Sin embargo, una manera de otorgarle vigor a la legislación medioambiental internacional que hasta ahora se maneja podría alcanzarse por medio de declarar al medio ambiente un sujeto de derecho, es decir, una ficción jurídica que concibe a la persona humana en su forma individual (persona natural) o colectiva (persona jurídica) como objeto de imputación de deberes y derechos ${ }^{8}$. Esto, de alguna manera, le otorga al medio ambiente personería jurídica y un valor intrínseco frente a las leyes. Sin embargo, detectamos un principal problema: la definición de sujeto de derecho, que concibe que solo son sujeto de derechos la persona humana en sus formas individual y colectiva. Con referencia a este punto, Bedón Garzón, citando a Slater, determina que los derechos son condición natural de los seres inteligentes como tales, que pueden reflexionar sobre sí mismos, conocer sus propias necesidades y que están capacitados para subvenir a ellas mediante la apropiación de objetos que le están subordinados y que pueden satisfacer aquellas necesidades. Esto nos lleva al reconocimiento de otro problema que deviene de la definición misma de sujeto de derecho, y es el hecho de que al ser objeto de imputación de derechos y de deberes, ¿cuáles serían entonces los deberes que se le deberían imputar a la naturaleza?

Otro fallo de esta postura radica en el ámbito netamente procesal del conflicto: si la naturaleza fuera sujeto de derecho y en, consecuencia, se le

7 Ley Especial que Regula el Otorgamiento de Concesiones Mineras en Áreas Urbanas y de Expansión Urbana.

8 Kelsen 1987: 93. 
otorgaran derechos y obligaciones, ¿cómo manifestaría su voluntad para ser defendida en los tribunales de nuestra sociedad? ¿Cuáles serían las obligaciones imputadas a la naturaleza? ¿Se le debería preguntar a un árbol o una vaca si desea ser cortado o sacrificada en beneficio del bienestar humano?

\section{El geocentrismo y otras maneras de proteger el medio ambiente}

La postura geocéntrica del derecho ambiental tiene sus antecedentes, para sorpresa de los autores del presente artículo, en el imperio del terror de la Alemania nazi. El nazismo concibió, durante su estadía en el poder, tres leyes que contemplaron las bases de lo que podría llamarse el ecologismo radical en la actualidad ${ }^{9}$. Sin embargo, no sería hasta la concepción de la Carta de la Naturaleza de las Naciones Unidas cuando se contempló que la especie humana es parte de la naturaleza y que la vida depende del funcionamiento ininterrumpido de los sistemas naturales, que son fuente de energía y materias nutritivas. Además, se reconoció que toda forma de vida es única y merece ser respetada, cualquiera que sea su utilidad para el hombre, y que, con el fin de reconocer a los demás seres vivos su valor intrínseco, el hombre debe guiarse por un código de acción moral ${ }^{10}$. A su vez, la ética ambiental biocéntrica, o geocentrismo, parte de considerar al hombre como un miembro más de la comunidad biosfera y no como un ser superior a las otras especies. Con ello, a la vez que establece que el hombre comparte un destino común con las otras especies y elementos de la Tierra, en tanto forma parte de su totalidad, invoca a un comportamiento ético de parte del hombre para con el medio ambiente.

Esta visión es posible gracias a los avances de la ciencia, en especial a la teoría de la evolución de Darwin, la cual contribuyó a concebir que la especie humana es solo una más del conjunto de seres que habitan la comunidad Tierra. La visión biocéntrica del hombre parte de este reconocimiento para establecer la identidad y el destino común que tiene el hombre con el resto de las especies ${ }^{11}$. Siguiendo esta línea de pensamiento, todos y cada uno de los componentes bióticos y abióticos de los ecosistemas tienen el derecho a existir y persistir por el valor que poseen, por el solo hecho de existir, ya sea porque nosotros nos beneficiemos de ellos o no.

Conscientes de esto, y respondiendo quizá a una necesidad de extender con mayor énfasis y rigurosidad la protección sobre su patrimonio natural (sus islas Galápagos fueron declaradas Patrimonio Natural de la

9 Serra 2003.

10 Carta Mundial de la Naturaleza.

11 lbarra 2009. 
Humanidad en 1979), Ecuador introdujo dentro de su constitución, cinco epígrafes que reconocen los derechos de la naturaleza, según los cuales la naturaleza tiene derecho a existir, persistir, mantener y regenerar sus ciclos vitales, estructura, funciones y procesos evolutivos. De igual forma, se reconoce el rol del Estado de aplicar medidas de precaución y restricción en todas las actividades que puedan conducir a la extinción de especies, la destrucción de los ecosistemas o la alteración permanente de los ciclos naturales y la introducción en los ecosistemas, de organismos y material orgánico e inorgánico que los puedan alterar de manera definitiva ${ }^{12}$. Se coloca así al hombre en la posición de proteger y velar por los derechos que no podían ser defendidos por la naturaleza misma, y se vence, en un principio, uno de los problemas que entrañaba declarar como sujeto de derecho a la naturaleza en el aspecto procesal.

Es en este punto en el que inevitablemente nos hacemos diferentes preguntas: ¿podría ser esto aplicable a nuestro país? ¿Significaría esto un retraso al desarrollo de nuestro país o, incluso, un agravio directo a los derechos de libre competencia de las empresas? ¿Es realmente justificable la pérdida permanente de toda nuestra biodiversidad por una mala praxis del Estado con fines meramente lucrativos? ¿Realmente el fin, el desarrollo económico del país, justifica los medios?

Es una respuesta negativa a la que llegamos como conclusión al final de cada una de aquellas preguntas. No se justifica, ni por todo el dinero del mundo, que se siga actuando de manera irresponsable e insensible con el medio ambiente, en una época tan importante y determinante como esta. Hemos llegado a un punto del camino en el que detenemos nuestro andar destructivo y aberrante, miramos hacia atrás y aceptamos todo lo que hemos hecho hasta ahora como base para estudiar los verdaderos impactos que hemos propiciado en el medio ambiente; para que, por medio de la aplicación de todo el conocimiento adquirido a lo largo de todos estos años, lleguemos a soluciones inteligentes y sostenibles para la preservación de la especie humana.

Como ejemplo de esto, encontramos que la Unión Europea lleva desarrollando políticas medioambientales muy estrictas desde hace muchos años para defender de manera efectiva el medio ambiente, así como Natura 2000 Network, red en defensa y cuidado del medio ambiente encargada de proteger más de 26 mil zonas verdes, pieza central de su Política de Naturaleza y Biodiversidad, que procura detener la extinción de especies y propicia el uso sostenible de recursos. Sin embargo, ¿qué tan sostenible y ecológico puede resultar crear islas ambientales, teniendo 
en consideración que todos los ecosistemas están interrelacionados y su desconexión podría devenir en una eventual pérdida de toda la biodiversidad? Eso, aunque preocupante y relevante para la humanidad, lastimosamente no forma parte de lo contemplado para el presente artículo.

Por otro lado, el Perú al ser considerado el tercer país a nivel mundial más vulnerable al cambio climático ${ }^{13}$, tiene la obligación de informar sobre su vulnerabilidad frente a este problema y presentar propuestas de adaptación necesarias para disminuir los impactos adversos producto del cambio climático. Nuestro país no está ajeno a esta realidad; como respuesta a ello, el 13 de mayo de 2008 se emitió el Decreto Legislativo 1013, que aprueba la creación del Ministerio del Ambiente (Minam), cuyo actual viceministro de Gestión Ambiental fue decano de la Facultad de Derecho de nuestra universidad y al cual han sido entregadas competencias como las de establecer la Política Nacional del Ambiente, la normatividad específica relevante para la materia, así como poseer funciones de administración, fiscalización y sanción en aras del cumplimiento de las normas ambientales ${ }^{14}$.

En ese sentido, el Minam tiene como finalidad ser un agente que funciona de manera sinérgica y transversal en todos los sectores relacionados con el medio ambiente, a fin de velar por una adecuada gestión ambiental. No obstante, el limitado poder que ostenta el Minam hasta el momento es, sin lugar a dudas, la más grande barrera que la efectiva tutela del medio ambiente enfrenta en nuestro país. Esto se puede ver reflejado en el hecho de que la aprobación de todos los estudios de impacto ambiental que se realizarán para llevar a cabo cualquier actividad —ya sea extractiva, productiva o de construcción y desarrollo- son aprobados por el ministerio de la competencia en cuestión (por ejemplo, en el caso de ser para minería, el Ministerio de Energía y Minas) y no por el Minam. Se observa así una irregular y deficiente situación en la cual una sola entidad juega el papel de juez y parte, lo cual resta seriedad, imparcialidad y credibilidad a las decisiones tomadas $y$, en consecuencia, propicia un aumento de la desconfianza de la sociedad, como se evidencia en el incremento de las tasas de conflictos socioambientales suscitados en toda la extensión de nuestro territorio.

Haciendo un cruce entre el análisis económico de derecho y el derecho ambiental basado en una concepción geocéntrica del hombre, nos damos cuenta de que no se está trabajando de manera adecuada

13 En http://radio.rpp.com.pe/cuidaelagua/peru-tercer-pais-mas-afectado-por-el-cambio-climatico. Consultado el 8 de julio de 2011.

14Decreto Legislativo 1013, que aprueba la Ley de Creación, Organización y Funciones del Ministerio del Ambiente. 
en el ámbito legislativo para la protección del medio ambiente, ya que podríamos de alguna manera intentar dar solución a la conflictiva de uso y abuso del medio ambiente, mediante la aplicación de titularidades con reglas de inalienabilidad, típicas del AED. Actualmente, y de acuerdo con Guido Calabresi, en Reglas de la propiedad, reglas de la responsabilidad e inalienabilidad: un vistazo a la catedral ${ }^{15}$, el medio ambiente es un bien, una propiedad que le pertenece al Estado y está administrado por medio de reglas de responsabilidad aplicadas en el sentido de que todo aquel que pretenda generar un impacto podría «comprar derechos para contaminar», toda vez que el beneficio que provea la actividad causante del daño es considerado suficiente para no reclamar su prohibición a nivel judicial; es decir, estar dispuesto a pagar un precio determinado por obtener un permiso que le permita contaminar, respetando los límites máximos permisibles.

En la práctica, esto degenera en que, a nivel administrativo, a muchas empresas les resulta mucho más económico pagar una multa por incurrir en una falta ambiental que invertir en evitarla, incluso cuando el daño causado fuera insostenible y generara efectos sobre el libre desarrollo de los derechos a la salud y un ambiente adecuado, ya antes mencionados como la forma más vinculante de defender al medio ambiente en un ámbito legal. El dilatado proceso que se iniciaría (como mínimo ocho años) tiene como resultado sanciones que muchas veces son insuficientes, si se las compara con el daño producido.

Por ello, como una probable solución a esta crisis, este equipo propone la institución de un ente rector internacional, especializado y capacitado adecuadamente en la gestión, administración y fiscalización del medio ambiente, que tenga las funciones de verificar que los protocolos, que hasta ahora han sido ratificados por todos y cada uno de los países adscritos, sean cumplidos; y que, en caso esto no suceda, ostente la fuerza necesaria para la aplicación de sanciones que traspasen los límites meramente morales y se materialicen en legislación vinculante con suficiente rigor sancionatorio, por medio de reglas de inalienabilidad aplicadas a la titularidad que recae sobre el medio ambiente y ostenta el Estado; se declara al Estado como una suerte de «incapaz» ${ }^{16}$, no considerando que el Estado sea incapaz de manejar sosteniblemente su patrimonio, sino más bien aludiendo al hecho de que el medio ambiente, al ser de relevancia

15 Calabresi y Melamed 1972.

16 En el estricto sentido legal de la palabra que se usa para definirlo, un incapaz es aquel individuo que, afecto de una incapacidad psíquica o física, es relevado de la capacidad de ejercicio que se adquiere al cumplir la mayoría de edad; se hace una analogía entre la capacidad de ejercicio del individuo y la del Estado. 
para la humanidad, debería ser administrado de manera conjunta y sinérgica por todos los Estados miembros de la comunidad mundial.

\section{Conclusiones}

Como parte de un grupo que ha interiorizado los conocimiento adquiridos en clase y de alguna manera los ha aplicado en el desarrollo del presente artículo, entendemos que los intereses monetarios y económicos en la actualidad van por delante del interés de preservar y cuidar los valiosos recursos naturales, para los cuales su categoría de agotable no está siendo considerada dentro de nuestro desarrollo económico. Reconocemos como grupo que sí son agotables y necesitan un tiempo prudente para su restitución natural. Se debe considerar también que, de mantenernos indiferentes frente a esta realidad, dentro de los próximos 30 años se padecerá de escasez de recursos alimenticios y, como si no ello fuera poco, del agotamiento de los mejores suelos del mundo.

Ratificamos también nuestra inclinación por la postura dogmática del geocentrismo moderado, que concibe que la naturaleza tiene el derecho de existir y regenerarse; se hace hincapié en que la prioridad para los humanos debería ser cuidar los ecosistemas existentes, toda vez que son necesarios para la supervivencia.

En nuestra opinión personal, Ecuador es un ejemplo a seguir en lo que a protección ambiental se refiere, al haber nombrado como sujeto de derecho a la naturaleza ecuatoriana. De este modo, Ecuador ha demostrado una postura de corte más eficiente frente a la crisis que significa el calentamiento global, así como saber cuidar y defender sus variados ecosistemas, en una decisión que en un futuro muy cercano les otorgará beneficios en diferentes aspectos económicos y sociales. No olvidemos que una de las primeras consecuencias que generaría la falta de recursos sería un incremento en su tasa de conflictos sociales.

Entendemos, a su vez, que las actividades económicas en nuestro país son fundamentalmente primarias. Por tanto, reconocemos que la implementación de medidas que promuevan el aprovechamiento sostenible de los recursos debe ser imperativa para el Perú. Más aún cuando los indicadores muestran que por mucho tiempo más deberemos depender de estos recursos, a causa de que no invertimos suficiente en investigación y desarrollo a efectos de hacer competitivas nuestras ventajas comparativas. El Perú invierte solo el $0,16 \%$ de su PBI en estos rubros, lo que representa 3,69 dólares por habitante al año. Otros países de la región como Brasil destinan el $0,82 \%$ del PBI (39,58 dólares por habitante); Chile, el 0,68\% (39,37 dólares por habitante), y Argentina, el 0,46\% (1.091 dólares por habitantes). En ese sentido, entendemos que nuestro país tiene que 
ampliar, con mayor énfasis, su actuar en los ámbitos educación e investigación, complementando así el aspecto social del desarrollo sostenible.

Hacemos también una recomendación con respecto al modo de proteger el medio ambiente: utilizando reglas de inalienabilidad, lo cual nos lleva a la reflexión inmediata de que esto tendría que darse bajo pena de afectar la soberanía de cada país, la cual, a nuestro parecer, debería sacrificarse en virtud y en beneficio de la supervivencia de la especie humana.

Actualmente, solo parecemos percibir las alzas o bajas extremas de las temperaturas. Es aquí donde hacemos un llamado de atención al Estado, a fin de que tome las medidas necesarias de difusión e información a la sociedad en su conjunto, para que se entienda el problema al que nos enfrentamos y colabore a cabalidad y convicción al participar de las medidas que se puedan adoptar para contrarrestar los efectos del cambio climático.

Concluimos, en total consenso, que esta es una época perfecta para vivir, ya que hemos decidido ver al cambio climático como una oportunidad para empezar a hacer las cosas mejor, considerar los efectos de lo que producimos (nuestra propia huella de carbono) y hacer todo lo posible para asegurarnos de que nuestro desarrollo sea sostenible para la generación presente como para las venideras. Este artículo nos ayuda en la tarea de informar y difundir más información con respecto a este tema.

\section{Agradecimientos}

Agradecemos a cada uno de nuestros padres, quienes, haciendo un inmenso esfuerzo para brindarnos un mejor futuro, nos han dado la oportunidad de estudiar en una universidad diferente, consciente de la importancia de ser empáticos con el medio ambiente y que propicia un espacio adecuado para el desarrollo y la formación de profesionales integrales, que no solo piensan en su desarrollo personal, sino en cómo este puede beneficiar a nuestra sociedad en una época tan decisiva como la que vivimos.

Asimismo, hacemos extensible un agradecimiento especial al magíster Héctor Aponte Ubillús, profesor y coordinador del curso de Ecología General de la Universidad Científica del Sur, curso dentro del cual adquirimos parte del conocimiento usado en el desarrollo del presente artículo y sin el cual su producción no hubiera sido posible, así como a la doctora Margarett Espinoza, profesora de la Facultad de Derecho de la universidad, quien participó como asesora y editora dentro del proceso de producción.

Por último, deseamos dedicar este artículo a los nietos de nuestros bisnietos, cuya inminente posibilidad de su existencia nos impulsa a hacer todo lo que se encuentre a nuestro alcance para preservar hoy el planeta que les cederemos mañana. 


\section{REFERENCIAS BIBLIOGRÁFICAS}

BARRANTES, Roxana (1993). Economía del medio ambiente. Consideraciones teóricas. Lima: Instituto de Estudios Peruanos.

BEDÓN, René (s. f.). «La naturaleza como sujeto de derechos. En http://www. uhemisferios.edu.ec/images/Imagenes_articulos/art.\%20ren\%20bedn.pdf. Consultado el 18 de enero de 2012.

CALABRESI, Guido y MELAMED, Douglas (1972). «Reglas de la propiedad, reglas de la responsabilidad e inalienabilidad: un vistazo a la catedral». En Harvard Law Review, nro. 6, vol. 85, pp. 1089-1128.

IBARRA, Guadalupe (2009). «Ética del medio ambiente». En http://www.elementos.buap.mx/num73/htm/11.htm. Consultado el 18 de enero de 2012.

KELSEN, Hans (1987). Teoría pura del derecho. Introducción a la ciencia del derecho. Buenos Aires: Editorial Eudeba.

KOONS, Jennifer (2008, setiembre). «LAW: Following Pa. mining town's example, Ecuador OKs constitution giving rights to nature». En http://www. celdf.org/article.php?id=185. Consultado el 18 de enero de 2012.

SERRA, Fernando (2003). «Otro punto de encuentro entre nazismo y marxismo. En http://www.ilustracionliberal.com/15/otro-punto-de-encuentro-entre-nazismo-y-marxismo-fernando-serra.html. Consultado el 18 de enero de 2012.

Recibido: Febrero de 2012.

Aceptado: Marzo de 2012. 